Case Study

\title{
The effects of pain scrambler therapy on the pain and quality of life of degenerative gonarthritis patients
}

\author{
Ho-Jin JeOng, $\mathrm{PT}^{1)}$, Dong-Kyu Lee, $\left.\mathrm{PT}^{2}\right)^{*}$, Yong-Nam Kim, PT, $\mathrm{PhD}^{3)}$, \\ Tae-Yeun Hwang, PT, PhD ${ }^{4}$ \\ 1) Department of Medical Sciences, Graduate School, Nambu University, Republic of Korea \\ 2) Department of Physical Therapy, Sunhan Hospital: 975 Mujin-daero, Seo-gu, Gwangju, \\ Republic of Korea \\ 3) Department of Physical Therapy, Nambu University, Republic of Korea \\ 4) Department of Physical Therapy, Chunnam Techno University, Republic of Korea
}

\begin{abstract}
Purpose] The purpose of this study was to identify the effects of pain scrambler therapy on the pain and quality of life of degenerative gonarthritis patients. [Subject and Methods] This study is a single case study. Pain scrambler therapy was applied for 40 minutes per session once a day for 15 days to a 62 -year-old female patient diagnosed with degenerative gonarthritis. Pain was measured using the Visual Analogue Scale and quality of life was measured using the short form-36 item after therapy. [Results] Decrease in pain and improved quality of life were evident after pain scrambler therapy. [Conclusion] Pain scrambler therapy had a potential to decrease pain and improve the quality of life in degenerative gonarthritis patients.

Key words: Pain scrambler therapy, Pain, Quality of life
\end{abstract}

(This article was submitted Sep. 22, 2017, and was accepted Dec. 19, 2017)

\section{INTRODUCTION}

Damage to cartilage cells in knee joints is due to various etiologies ${ }^{1)}$. Degenerative arthritis starts as the ligaments and muscles moving the joints are weakened and the cartilage is worn out, leading to pain, ankyloses, decrease in the range of joint movement, and edema as the main presenting symptoms ${ }^{1,2)}$. Degenerative gonarthritis is a disease that causes physical problems such as functional disorder and pain as well as a decrease in the quality of life, causing psychosocial problems ${ }^{2)}$. Based on information theory, pain scrambler therapy transmits artificially created analgesia nerve information to the wrong algesthesis nerve system of the brain, which causes chronic pain ${ }^{3,4)}$. Pain scrambler therapy was developed as an alternative to existing drug regimens, injections, and surgery as a new treatment for pain in patients with intractable chronic pain or cancer pain ${ }^{4}$. The purpose of this study was to identify the effects of pain scrambler therapy on pain and quality of life in degenerative gonarthritis patients.

\section{SUBJECT AND METHODS}

This study is a single case study. The study was conducted on a 62-year-old female patient with degenerative gonarthritis. The subject had no issues with her blood pressure, pulse, respiration, consciousness, or senses. The subject agreed with the purpose and methods of the study prior to its commencement. The study adhered to the principles of the Declaration of Helsinki. Pain scrambler therapy was conducted by attaching electrode-special types to areas $2-3 \mathrm{~cm}$ away from parts with

*Corresponding author. Dong-Kyu Lee (E-mail: ldkpt@hanmail.net)

(C2018 The Society of Physical Therapy Science. Published by IPEC Inc.

(c) $(-)$ This is an open-access article distributed under the terms of the Creative Commons Attribution Non-Commercial No Deriva-

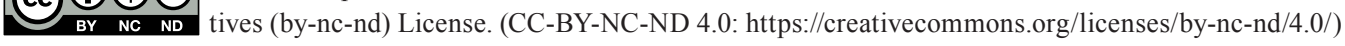


severe pain using 5 channels. Frequency was set at $43-52 \mathrm{~Hz}$ and the stimulus strength was set at $5 \mathrm{~mA}$ to generate natural electric signal transmitted to her body. There were 16 analgesia waveforms built in the pain scrambler. The therapy was applied for 40 minutes a session once for a total of 15 days. Pain was measured using the Visual Analogue Scale (VAS). The VAS is a self-reported pain scale that asks the patient to mark the degree of pain ranging from 0 to 10 , with higher scores indicating more pain. Quality of life was measured using short form 36-item (SF-36), which consists of 8 items in 36 questions on physical health, such as physical function, limitations on physical roles, pain, and general health and mental health, comprised of vigor, social function, limitations on emotional roles, and mental health. A higher score is indicative of better quality of life.

\section{RESULTS}

The VAS score decreased from 9 to 1 after the pain scrambler therapy. Scores on SF-36 increased to 95 from 79 prior to pain scrambler therapy.

\section{DISCUSSION}

The purpose of this study was to identify the effects of pain scrambler therapy on pain and quality of life of degenerative gonarthritis patients. The application of pain scrambler therapy on a degenerative gonarthritis patient showed that pain decreased and quality of life improved. Pain scrambler therapy encodes the analgesia information system and recovers autonomic neuro-regulation function of the brain through the algesthesis nerve route ${ }^{4}$. Drug therapy, injection, and surgical treatment make algesthesis work in the central nervous system to block the route of transmission to the brain, stimulating the A-beta nerve and preventing pain ${ }^{5}$. On the contrary, pain scrambler therapy naturally affects the analgesia signal system of A-delta and C-fibers instead of blocking the pain routes ${ }^{5)}$. The frequency of pain scrambler therapy is about $43-52 \mathrm{~Hz}$ and the stimulus strength is $5 \mathrm{~mA}$, which generates the artificial neuronal information to be transferred percutaneously in a wavelength signal form to the algesthesis nerve through the body ${ }^{3-5}$. Pain scrambler therapy has effectively decreased pain such as chronic pain, intractable pain, cancer pain, and neuropathy pain by autonomically recovering neuro-regulation functions $^{4,5)}$. Many different studies have reported that a decrease in pain also influences the quality of life ${ }^{6,7)}$. Results indicate that pain scrambler therapy has a positive effect in decreasing the pain and improving the quality of life in degenerative gonarthritis patients. The study is limited by its small sample size, which affects generalizability of results. A follow-up study was not conducted to examine the long-term effects of pain scrambler therapy. A long-term study should be conducted on degenerative gonarthritis patients, with larger sample sizes for increased generalizability.

\section{Conflict of interest}

None.

\section{REFERENCES}

1) Davies CM, Guilak F, Weinberg JB, et al.: Reactive nitrogen and oxygen species in interleukin-1-mediated DNA damage associated with osteoarthritis. Osteoarthritis Cartilage, 2008, 16: 624-630. [Medline] [CrossRef]

2) Turner JA, Ersek M, Kemp C: Self-efficacy for managing pain is associated with disability, depression, and pain coping among retirement community residents with chronic pain. J Pain, 2005, 6: 471-479. [Medline] [CrossRef]

3) Marineo G: Untreatable pain resulting from abdominal cancer: new hope from biophysics? JOP, 2003, 4: 1-10. [Medline]

4) Sabato AF, Marineo G, Gatti A: Scrambler therapy. Minerva Anestesiol, 2005, 71: 479-482. [Medline]

5) Smith TJ, Coyne PJ, Parker GL, et al.: Pilot trial of a patient-specific cutaneous electrostimulation device (MC5-A Calmare $\left.{ }^{\circledR}\right)$ for chemotherapy-induced peripheral neuropathy. J Pain Symptom Manage, 2010, 40: 883-891. [Medline] [CrossRef]

6) Lowry V, Ouellet P, Vendittoli PA, et al.: Determinants of pain, disability, health-related quality of life and physical performance in patients with knee osteoarthritis awaiting total joint arthroplasty. Disabil Rehabil, 2017, 20: 1-11. [Medline] [CrossRef]

7) Mesci E, Icagasioglu A, Mesci N, et al.: Relation of physical activity level with quality of life, sleep and depression in patients with knee osteoarthritis. N Clin Istanb, 2015, 2: 215-221. [Medline] 